\title{
Long-time self-diffusion of Brownian Gaussian-core particles
}

\author{
H. H. Wensink, H. Löwen, M. Rex, C. N. Likos and S. van Teeffelen \\ Institut für Theoretische Physik II: Weiche Materie, \\ Heinrich-Heine-Universität Düsseldorf, Universitätsstraße 1, D-40225 Düsseldorf, Germany
}

(Dated: October 26, 2018)

\begin{abstract}
Using extensive Brownian dynamics computer simulations, the long-time self-diffusion coefficient is calculated for Gaussian-core particles as a function of the number density. Both spherical and rodlike particles interacting via Gaussian segments are considered. For increasing concentration we find that the translational self-diffusion behaves non-monotonically reflecting the structural reentrance effect in the equilibrium phase diagram. Both in the limits of zero and infinite concentration, it approaches its short-time value. The microscopic Medina-Noyola theory qualitatively accounts for the translational long-time diffusion. The long-time orientational diffusion coefficient for Gaussian rods, on the other hand, remains very close to its short-time counterpart for any density. Some implications of the weak translation-rotation coupling for ultrasoft rods are discussed.
\end{abstract}

PACS numbers: 66.10.Cb; 61.20.Ja; 82.70.Dd

\section{INTRODUCTION}

Particles interacting via penetrable pair potentials exhibit fascinating new clustering and reentrance effects [1, 2, [3, 4, 5] which are absent for diverging potentials such as hard spheres and inverse-power potentials. A well-studied model for a penetrable interaction is a Gaussian potential [3, 6, 7, 8, [9] which mimics the effective interactions between two polymer coils in a good solvent [10] and applies also to dendrimer solutions [11, 12, 13]. This potential can be generalized towards a Gaussiansegment model for rod-like particles in order to describe bottlebrush polymers with a stiff backbone 14], see also [15]. In the Gaussian-core system, two particles pay a finite energy penalty if they are sitting on top of each other. If they overlap completely there is no repulsive force any longer.

In the present paper we focus on equilibrium dynamical correlations of Gaussian Brownian fluids. In particular the long-time self-diffusion coefficient is simulated as a function of the particle density. Recently the dynamical behavior of spherical Gaussian particles has been explored by molecular dynamics studies [16] which is suitable for polymer melts but neglects the hydrodynamic friction of a solvent. Here, we consider solutions of colloidal or polymeric particles and therefore overdamped Brownian dynamics is appropriate where the friction of the solvent is included. We further study the long-time translational and orientational self-diffusion of Gaussian segment rods in the isotropic phase as a function of rod concentration.

As a result, we find that the long-time self-diffusion coefficient behaves non-monotonically with density, both for spheres and rods. For zero density (i.e. the single particle limit) the long-time self-diffusion coefficient is clearly identical to the short-time diffusion constant which is entirely dominated by solvent friction. What is less obvious is that for high densities with multiple overlap of particles the self-diffusion again tends to its shorttime counterpart. In fact in the limit of very high densi- ties, a Gaussian particle feels many neighbors around a distance where the Gaussian potential has its inflection point and these give rise to a diverging number of interaction kicks. Therefore one could have expected a higher diffusion coefficient than the short-time value. However, we show that correlations between the neighboring particles enforce a normal diffusive behavior of an effective ideal gas in this limit.

Between these two extreme limits, for finite densities, the long-time self-diffusion coefficient is smaller than its short-time counterpart. The minimal value is roughly at the point of maximal fluid structure. A similar nonmonotonic behavior has been found for molecular dynamics [16] where the ballistic limit of zero-density leads, of course, to a diverging long-time self-diffusion coefficient. Furthermore we find that the long-time orientational selfdiffusion coefficient practically coincides with its shorttime behavior. The latter fact implies that there is no significant translation-rotation coupling in the Gaussian segment model for penetrable rods.

We compare our simulation data with the microscopic theory of Medina-Noyola [17, 18] which relates the longtime self-diffusion coefficient to the fluid pair correlation and find qualitative agreement for spheres. The same holds for the translational diffusion of rods if the theory of Medina-Noyola [17] is applied to the translational degrees of freedom alone.

The paper is organized as follows: in Section II we describe in detail the procedure of the Brownian dynamics computer simulation. Results for the long-time self diffusion are presented and discussed in Section III. Finally Section IV is devoted to more general remarks and conclusions.

\section{BROWNIAN DYNAMICS COMPUTER SIMULATIONS}

The Brownian dynamics (BD) simulations are based on a finite-difference integration of the overdamped 
Langevin equations for $N$ interacting anisometric particles in three dimensions [19, 20]. The trajectory of each particle $i$ is characterized by its position $\mathbf{r}_{i}(t)$ and orientation $\hat{\omega}_{i}(t)$ at time $t$. If hydrodynamic interactions are neglected, the update equation for the position of particle $i$ can be written in the following way

$$
\mathbf{r}_{i}(t+\Delta t)=\mathbf{r}_{i}(t)+\frac{\Delta t}{k_{B} T} \mathbf{D}_{0}^{T} \cdot \mathbf{F}_{i}(t)+\Delta \mathbf{r}_{i}+\mathcal{O}\left\{(\Delta t)^{2}\right\},
$$

with $k_{B} T$ the thermal energy and $\mathbf{F}_{i}(t)$ the total force acting on the center-of-mass. The latter is derived from the pair potential which will be specified later. Furthermore, $\mathbf{D}_{0}^{T}$ represents the short-time diffusion tensor which in case of uniaxially symmetric particles (e.g. cylinders) can be cast into the form

$$
\mathbf{D}_{0}^{T}=D_{0}^{\|}\left(\hat{\omega}_{i} \otimes \hat{\omega}_{i}\right)+D_{0}^{\perp}\left(\hat{\mathbf{I}}-\hat{\omega}_{i} \otimes \hat{\omega}_{i}\right),
$$

in terms of the translational diffusion coefficients parallel $\left(D_{0}^{\|}\right)$and perpendicular $\left(D_{0}^{\perp}\right)$ to the particle axis, with $\hat{\mathbf{I}}$ the unit tensor and $\otimes$ a dyadic product.

The contribution $\Delta \mathbf{r}_{i}$ denotes a random displacement of the particle due to collisions with the solvent molecules. Similar to Eq. (2), it is convenient to decompose it into contributions parallel and perpendicular to the particle axis. Introducing two orthogonal unit vectors, $\hat{e}_{1 i}$ and $\hat{e}_{2 i}$, perpendicular to $\hat{\omega}_{i}$ we can express the noise term in Eq. (11) as

$$
\Delta \mathbf{r}_{i}=\Delta r^{\|} \hat{\omega}_{i}(t)+\Delta r_{(1)}^{\perp} \hat{e}_{1 i}(t)+\Delta r_{(2)}^{\perp} \hat{e}_{2 i}(t) .
$$

Here, $\Delta r \|$ and $\Delta r_{(1,2)}^{\perp}$ represent Gaussian random displacements parallel and perpendicular to the symmetry axis. Both stochastic quantities have zero mean and their variance is $2 D_{0}^{\|} \Delta t$ and $2 D_{0}^{\perp} \Delta t$, respectively.

The orientational update equation for $\hat{\omega}_{i}(t)$ reads

$\hat{\omega}_{i}(t+\Delta t)=\hat{\omega}_{i}(t)+\frac{\Delta t}{k_{B} T} D_{0}^{R} \mathbf{T}_{i}(t) \times \hat{\omega}_{i}(t)+\Delta \hat{\omega}_{i}+\mathcal{O}\left\{(\Delta t)^{2}\right\}$.

Here, $D_{0}^{R}$ denotes the short-time rotational diffusion coefficient and $\mathbf{T}_{i}(t)$ the total center-of-mass torque acting on particle $i$. The noise contribution,

$$
\Delta \hat{\omega}_{i}=x_{1} \hat{e}_{1 i}(t)+x_{2} \hat{e}_{2 i}(t),
$$

is generated by means of two uncorrelated random Gaussian numbers, $x_{1}$ and $x_{2}$, both with zero mean and variance $2 D_{0}^{R} \Delta t$. After each step the new orientations $\hat{\omega}_{i}(t+\Delta t)$ have to be renormalized to ensure that $\left|\hat{\omega}_{i}\right|=1$ at all times.

Obviously, for spherical particles the translational Brownian motion is completely decoupled from the orientations and the translational diffusion tensor Eq. (2) becomes diagonal, i.e. $\mathbf{D}_{0}^{T}=D_{0}^{T} \hat{\mathbf{I}}$. In this case, we need only consider the update equation for the positional coordinates Eq. (11). All update equations are exact up

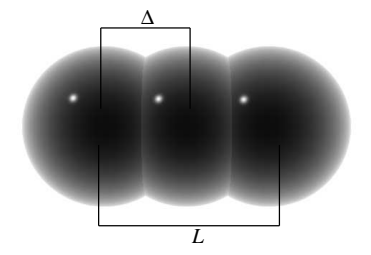

FIG. 1: Soft rod of length $L$ composed of $N_{S}=3$ segments with intersegment distance $\Delta$.

to order $\mathcal{O}(\Delta t)$ which suffices for the present purpose, provided $\Delta t$ is chosen small enough. For a detailed discussion of a second order update algorithm the reader is referred to Ref. [20].

The pair potential of the particles is given by an ultrasoft Gaussian potential. For spherically symmetric particles we have:

$$
v_{2}(r)=\epsilon \exp \left[-(r / \sigma)^{2}\right]
$$

where $\sigma$ is the potential range which will henceforth serve as our unit of length. The amplitude is fixed at $\epsilon=5 k_{B} T$. According to [3], the associated reduced temperature $T^{*}=k_{B} T / \epsilon=0.2$ is much higher than the upper freezing temperature $T^{*} \cong 0.01$ which guarantees a stable fluid state at any density.

Apart from ultrasoft spheres we will also consider systems of Gaussian rods [14]. Each rod has length $L$ and is composed of $N_{S}$ spherical segments placed at equidistant positions along the rod main axis with segment-segment distance $\Delta=L /\left(N_{S}-1\right)$. The interaction potential between two segments from different rods is again a Gaussian. The pair potential between two rods $i$ and $j$ is then given by a sum over all segment interactions:

$$
v_{2}\left(\mathbf{r}_{i}, \mathbf{r}_{j} ; \hat{\omega}_{i}, \hat{\omega}_{j}\right)=\epsilon \sum_{\alpha=-K}^{K} \sum_{\beta=-K}^{K} \exp \left[-\left(\left|\mathbf{r}_{\alpha \beta}\right| / \sigma\right)^{2}\right],
$$

where $K=\left(N_{\mathrm{S}}-1\right) / 2$ and $\mathbf{r}_{\alpha \beta}=\left(\mathbf{r}_{i}+\alpha \Delta \hat{\omega}_{i}\right)-\left(\mathbf{r}_{j}+\right.$ $\left.\beta \Delta \hat{\omega}_{j}\right)$ the distance between segment $\alpha$ on $\operatorname{rod} i$ and $\beta$ on $\operatorname{rod} j(i \neq j)$. We will consider slightly anisometric rods with $N_{\mathrm{S}}=3$ segments and $L=2 \sigma$ (see Fig. 1 for a sketch). Furthermore the segment-segment potential amplitude is $\epsilon=5 k_{B} T$. The short-time diffusion coefficients of the rods depend on one-particle hydrodynamic effects. For these, we take the analytical results obtained for hard ellipsoids of length $L$ and aspect-ratio $p>1$ reported by Tirado and co-workers [21]:

$$
\begin{aligned}
D_{0}^{\perp} & =\frac{3 D_{0}^{T}}{2 p}\left(\ln p+0.839+0.185 / p+0.233 / p^{2}\right), \quad(8) \\
D_{0}^{\|} & =\frac{3 D_{0}^{T}}{p}\left(\ln p-0.207+0.980 / p-0.133 / p^{2}\right), \quad(9) \\
D_{0}^{R} & =\frac{18}{\pi p^{3}} \frac{D_{0}^{T}}{\sigma^{2}}\left(\ln p-0.662+0.917 / p-0.050 / p^{2} \gamma 10\right)
\end{aligned}
$$

with $D_{0}^{T}=k_{B} T / 6 \pi \eta_{s} \sigma$ the short-time diffusion constant of a sphere with radius $\sigma$ and $\eta_{s}$ the shear viscosity of the solvent. In the above, we have implicitly 
identified $L=p \sigma$, with $p$ the hydrodynamic aspect-ratio of the Gaussian rods. In order to enforce a substantial translation-rotation coupling we take a value $p=5$ which is larger than the interaction aspect-ratio $L / \sigma=2$.

A natural unit of time is the Brownian time $\tau_{B}=$ $\sigma^{2} / D_{0}^{T}$ defined as the typical time a Gaussian particle needs to diffuse over a distance comparable to its own dimension. Let us further introduce $\bar{D}_{0}^{T}=\left\|\oint d \hat{\omega} \mathbf{D}_{i}^{T}\right\|$, the isotropic orientational average of the diffusion tensor Eq. (2). For the spheres $\bar{D}_{0}^{T}=D_{0}^{T}$ while for the rods

$$
\bar{D}_{0}^{T}=\frac{1}{3} D_{0}^{\|}+\frac{2}{3} D_{0}^{\perp} .
$$

With this result, we may compute the ratio of the singlerod rotational and translational relaxation times, i.e. $\tau_{0}^{R} / \tau_{0}^{T}=\bar{D}_{0}^{T} / p^{2} \sigma^{2} D_{0}^{R} \cong 0.264$, showing that the shorttime orientational dynamics is much faster than the translational. The quantity $\bar{D}_{0}^{T}$ also provides the natural scale for the long-time translational self-diffusion coefficient $D_{L}^{T}$, defined as

$$
D_{L}^{T}=\lim _{t \rightarrow \infty} \frac{1}{6 t} \frac{1}{N}\left\langle\sum_{i=1}^{N}\left(\mathbf{r}_{i}(t)-\mathbf{r}_{i}(0)\right)^{2}\right\rangle,
$$

where $\langle\cdots\rangle$ is a canonical average. An alternative definition is provided by a differential expression

$$
D_{L}^{T}=\lim _{t \rightarrow \infty} \frac{1}{6} \frac{d}{d t} \frac{1}{N}\left\langle\sum_{i=1}^{N}\left(\mathbf{r}_{i}(t)-\mathbf{r}_{i}(0)\right)^{2}\right\rangle .
$$

Both expressions should in principle yield identical results in the long-time limit. In practice however, they will differ slightly and the difference can be used to assess the error in $D_{I}^{T}$.

Following Ref. [20] we can define the long-time rotational diffusion coefficient $D_{L}^{R}$ as follows

$$
D_{L}^{R}=-\lim _{t \rightarrow \infty} \frac{W_{n}(t)}{t}
$$

where $W_{n}(t)$ is an orientational correlation function measuring the mean-square displacement on the unit sphere. It is given by

$$
W_{n}(t)=\frac{1}{n(n+1)} \ln \left\langle\mathcal{P}_{n}(\hat{\omega}(t) \cdot \hat{\omega}(0))\right\rangle,
$$

with $\mathcal{P}_{n}$ a Legendre polynomial. Similar to Eq. (13), we may also employ the differential analogue. If the rotational motion is a diffusion process on the unit sphere, the dynamics is captured by the Debye diffusion equation which predicts $D_{L}^{R}$ to be independent of $n$ [20].

In our simulations we used a cubic simulation box of volume $V$ with periodic boundary conditions in all three directions. The number of particles depends on the number density. If we choose to cut off all segment-segment pair interaction for which $v_{2} / \epsilon<v_{2}^{\text {cut }} / \epsilon$ the length $L_{B}$ of the simulation box must be at least twice the corresponding cutoff range and so $L_{B} / \sigma>-2 \ln \left[v_{2}^{\text {cut }} / \epsilon\right]$.

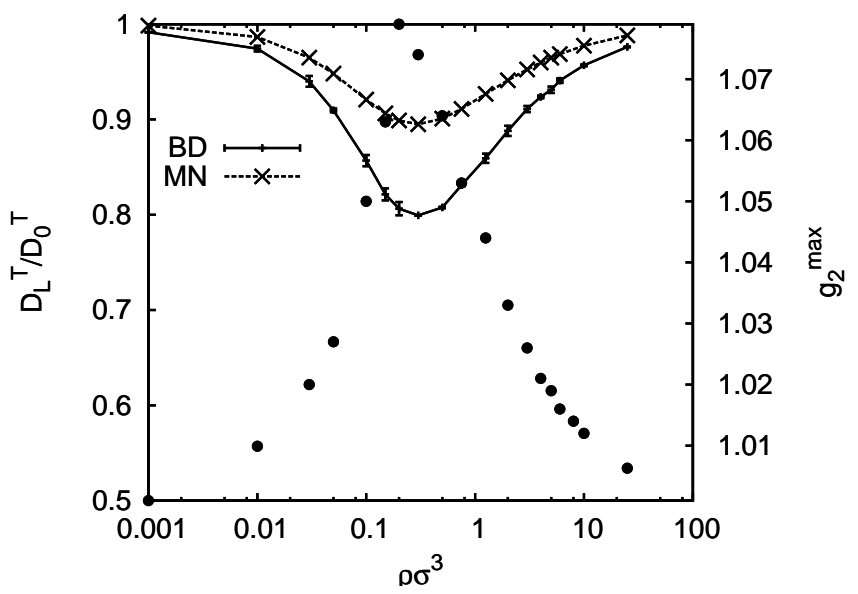

FIG. 2: Long-time translational self-diffusion coefficient divided by its low-density limit $D_{L}^{T} / D_{0}^{T}$ versus the density $\rho \sigma^{3}$ (on a $\log$ scale) for Gaussian spheres from BD simulations and the theory of Medina-Noyola (MN). On the right vertical axis, filled dots give the amplitude $g_{2}^{\max }$ of the first maximum of the equilibrium pair correlation function $g_{2}(r)$.

For a given density the number of particles must therefore obey $N>\left(\rho \sigma^{3}\right) L_{B}^{3}$, while imposing a minimum of $N=500$ at small densities. Using $v_{2}^{\text {cut }} / \epsilon=10^{-6}$, a run at $\rho \sigma^{3}=10$ requires $N=4000$ and one at $\rho \sigma^{3}=25$ $N=13000$. The time step must be reasonably small and was fixed at $0.0005 \tau_{B}$. Initial configurations were generated by putting the particles at random positions. For the rod systems, a parallel nematic initial configuration was adopted. After a long equilibration period of at least $10 \tau_{B}$ statistics were gathered (during a period of about $15 \tau_{B}$ ) and the time-dependent correlations were monitored during an interval of about $5 \tau_{B}$. The latter turned out to be sufficiently large to reach the long-time limit.

\section{RESULTS FOR THE LONG-TIME SELF-DIFFUSION COEFFICIENTS}

Results for the long-time diffusion coefficient for spheres are shown in Fig. 2. A clear non-monotonic behavior is observed. Both for very small and very high densities, the diffusivity comes very close to that of a single particle. At the highest density simulated $\left(\rho \sigma^{3}=25\right)$ the long-time diffusion constant has regained about 98 $\%$ of its short-time value indicating that the diffusion has become virtually ideal in the high-density limit. The density $\rho \sigma^{3} \cong 0.3$ at which the diffusivity becomes minimal is in agreement with the results of Ref. [16], and lies close to the density $\rho \sigma^{3} \cong 0.23$ for which the Gaussian core model displays its 'turning point' in the reentrant melting transition [3].

To compare our data with microscopic theory we have included the prediction from the Medina-Noyola theory for self-diffusion [17]. The theory comprises an analysis of the effective Langevin equation of a tagged spherical 


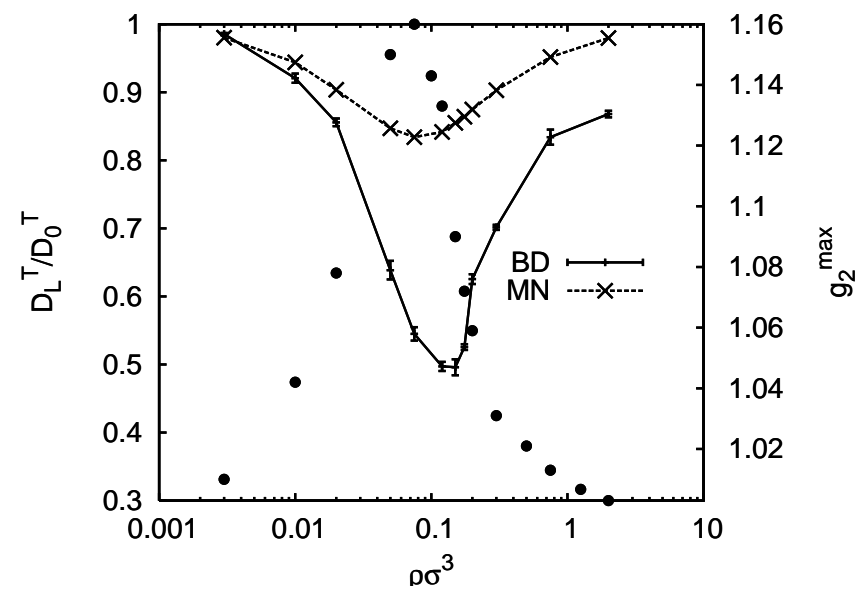

FIG. 3: Same as Fig. 2 for the Gaussian segment rods.

colloid in a medium of interacting neighbor particles. In the absence of hydrodynamic interactions, the following expression for $D_{L}^{T}$ is proposed:

$$
D_{L}^{T}=D_{0}^{T}\left(1+\frac{\rho}{6} \int d \mathbf{r}\left[g_{2}(r)-1\right]^{2}\right)^{-1},
$$

where the only input is the static pair correlation function $g_{2}(r)$ which is obtained from the simulation. Although the theory is certainly not reliable from a quantitative point of view, as we observe in Fig. 2, the nonmonotonic behavior is clearly recovered. This suggests that there is a qualitative correspondence between the long-time diffusive behavior and the static correlations as embedded in $g_{2}(r)$. This becomes more explicit when we compare the diffusion data with the maximum amplitude in the pair correlation function, also shown in Fig. 2, The behavior for the rods is qualitatively the same as for spheres, see Fig. 3 Also here the translational diffusion constant varies non-monotonically with density and approaches the short-time limits at small and large densities. The only notable difference is that the fluid structure is somewhat more pronounced here. As a result, the normalized diffusion constant reaches a minimum value that is smaller than that for spheres. Again, the theory of Medina-Noyola now taken with the center-of-mass pair correlations as an input overestimates the simulation data but shows the correct trend.

Contrary to $D_{L}^{T}$, the rotational counterpart in Fig. 4 seems to be weakly affected by the density. At the point of maximum fluid structure $\left(\rho \sigma^{3} \cong 0.12\right)$ the long-time rotational diffusivity has dropped to only about $90 \%$ of the maximum i.e. short-time value. Moreover, an investigation of $W_{n}(t)$ at minimum diffusivity shows that the mean-square orientational displacement does not depend on $n$. From this we may conclude that the rotational relaxation on the unit sphere is purely diffusive for long times. This behavior is not found in isotropic systems with unbounded rod potentials such as hard spherocylinders or Yukawa segment models [20]. The distinct dis-
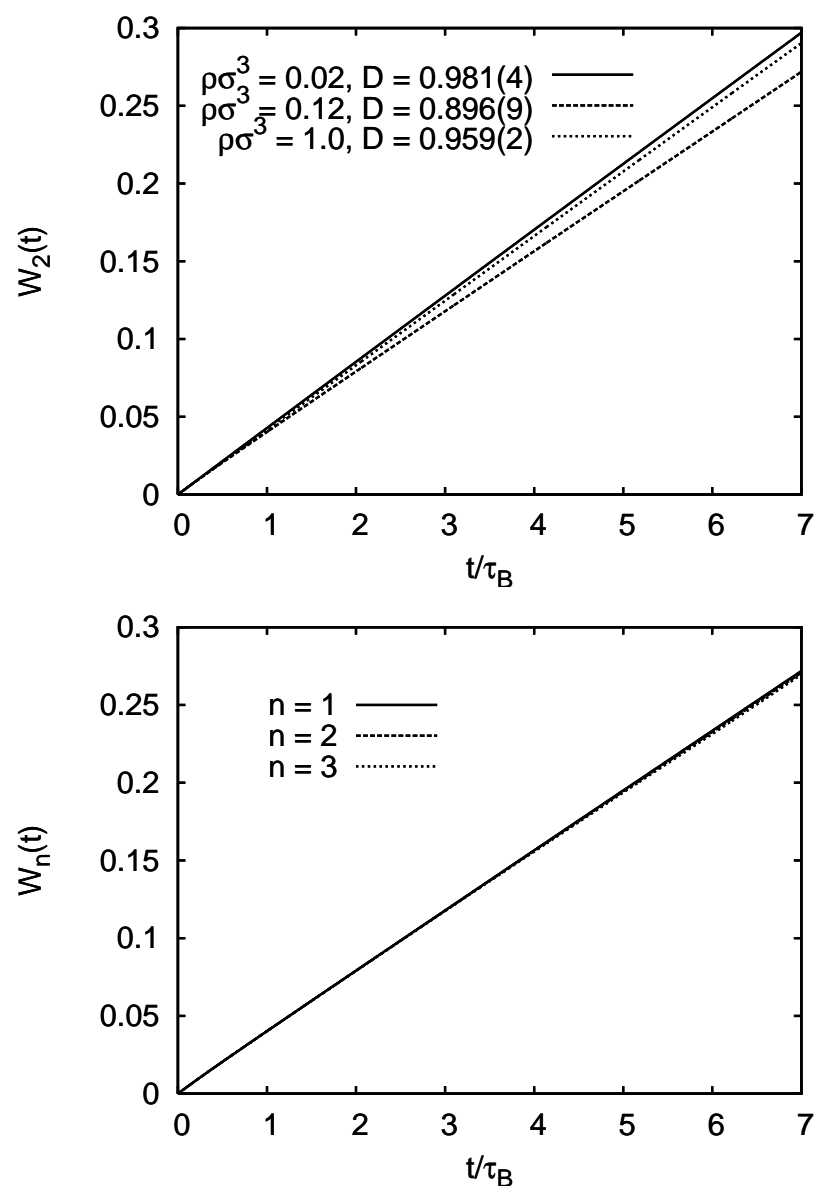

FIG. 4: (top) Mean-square displacement on the unit spheres $W_{2}(t)$ [see Eq. (15)] for Gaussian rods at various densities. The corresponding long-time rotational diffusion coefficients $D=D_{L}^{R} / D_{0}^{R}$ are indicated where the number in brackets gives the error of the last digit. (bottom) $W_{n}(t)$ for $n=1,2,3$ for the system with density $\rho \sigma^{3}=0.12$.

crepancy between the density-dependence of the translational and rotational diffusivity suggests that the coupling between orientational and translational degrees of freedom is very small for the systems of ultrasoft rods considered here.

Some insight as to the status of the translation-rotation coupling can be gained by considering the effective interaction of a rod pair in a spatially homogeneous fluid:

$$
v_{2}\left(\hat{\omega}_{i}, \hat{\omega}_{j}\right)=\int d \mathbf{r}_{i j} v_{2}\left(\mathbf{r}_{i j} ; \hat{\omega}_{i}, \hat{\omega}_{j}\right),
$$

with $\mathbf{r}_{i j}=\mathbf{r}_{j}-\mathbf{r}_{i}$. Inserting Eq. (7) and some algebra leads to $v_{2}\left(\hat{\omega}_{i}, \hat{\omega}_{j}\right)=$ const. This result holds for any bounded segment-segment potential and implies that, irrespective of the rod aspect-ratio, all static orientational correlations are rendered zero by the random-phase approximation for the excess free energy [2, 3]. For a spatially uniform rod fluid the latter is simply proportional to a double orientational average of $v_{2}\left(\hat{\omega}_{i}, \hat{\omega}_{j}\right)$. This will give the same outcome for any normalized orientational 
distribution. Since the ideal free energy of a nematic fluid is always higher than that of the isotropic, the possibility of a stable nematic state is fully excluded. Of course the above argument does not rule out a possible freezing transition occurring within an isotropic rod fluid. In fact, recent investigations for other soft rods such as parallel Gaussian-core particles [15] and Yukawa rods [22] seem to point to a pronounced stability of columnar liquidcrystalline order in these systems. Finally, we remark that for soft rods with large aspect-ratios a phase transition from an isotropic toward a nematic fluid may be possible at low densities where rod correlations are much better described by the Onsager functional [23] than the random-phase approximation.

\section{CONCLUSIONS}

In conclusion, we have simulated the long-time selfdiffusion in concentrated Brownian systems of rod-like and spherical particles which interact via a Gaussian core and are thus penetrable. As reflected by the statics, the system is getting ideal in the high-density limit where the random-phase approximation for the fluid structure becomes asymptotically exact. We think that the trends are independent of details in the interaction potentials provided that clustering [2] is avoided.

We finish with a few remarks. First of all, one should consider the hydrodynamic interactions mediated by the solvent. These are neglected in the Brownian dynamics simulations, but can be treated using more sophisticated (and time-consuming) schemes like latticeBoltzmann, Stokesian or the stochastic rotation dynamics [24, 25, 26, 27, 28]. Second, there is a need to derive microscopic models for the Brownian motion of stiff rods with soft interactions on the basis of the Smoluchowski equation involving mode-coupling approximations. These approaches then would go beyond a simple effective Medina-Noyola theory in treating explicitly the orientational degrees of freedom. Our simulation results may provide benchmark data to test these theories. As to the statics, it would be worthwhile to map out the freezing behavior of Gaussian segment rods in the regime of high density and low temperature. Finally, the existence of a stable nematic phase for Gaussian rods with large aspect-ratio remains an open question.

\section{Acknowledgments}

Financial support from the Deutsche Forschungsgemeinschaft under SFB-TR6 is gratefully acknowledged.
[1] C. N. Likos, B. M. Mladek, A. J. Moreno, D. Gottwald, and G. Kahl, to be published (2007).

[2] C. N. Likos, A. Lang, M. Watzlawek, and H. Löwen, Phys. Rev. E 63, 031206 (2001).

[3] A. Lang, C. N. Likos, M. Watzlawek, and H. Löwen, J. Phys.: Condens. Matter 12, 5087 (2000).

[4] B. M. Mladek, D. Gottwald, G. Kahl, M. Neumann, and C. N. Likos, Phys. Rev. Lett. 96, 045701 (2006).

[5] D. Frenkel, Science 314, 768 (2006).

[6] F. H. Stillinger, J. Chem. Phys. 65, 3968 (1976).

[7] F. H. Stillinger and T. A. Weber, J. Chem. Phys. 70, 4879 (1979).

[8] A. A. Louis, P. G. Bolhuis, and J. P. Hansen, Phys. Rev. E 62, 7961 (2000).

[9] S. Prestipino, F. Saija, and P. V. Giaquinta, Phys. Rev. E 71, 050102 (2005).

[10] A. A. Louis, P. G. Bolhuis, and J. P. Hansen, Phys. Rev. Lett. 85, 2522 (2000).

[11] C. N. Likos, M. Schmidt, H. Löwen, M. Ballauff, D. Pötschke, and P. Lindner, Macromolecules 34, 2914 (2001).

[12] C. N. Likos, S. Rosenfeldt, N. Dingenouts, M. Ballauff, P. Lindner, N. Werner, and F. Vögtle, J. Chem. Phys. 117, 1869 (2002).

[13] I. O. Götze, H. M. Harreis, and C. N. Likos, J. Chem. Phys. 120, 7761 (2004).
[14] M. Rex, H. H. Wensink, and H. Löwen, Phys. Rev. E 76, 021403 (2007).

[15] S. Prestipino and F. Saija, J. Chem. Phys. 126, 194902 (2007).

[16] P. Mausbach and H. O. May, Fluid Phase Eq. 249, 17 (2006).

[17] M. Medina-Noyola, Phys. Rev. Lett. 60, 2705 (1988).

[18] H. Löwen and G. Szamel, J. Phys.: Condens. Matter 5, 2295 (1993).

[19] H. Löwen, Phys. Rev. E 50, 1232 (1994).

[20] T. Kirchhoff, H. Löwen, and R. Klein, Phys. Rev. E 53, 5011 (1996).

[21] M. M. Tirado, J. G. de la Torre, and C. L. Martinez, J. Chem. Phys. 81, 2047 (1984).

[22] H. H. Wensink, J. Chem. Phys. 126, 194901 (2007).

[23] L. Onsager, Ann. N.Y. Acad. Sci. 51, 627 (1949).

[24] L. Durlofsky, J. F. Brady, and G. Bossis, J. Fluid Mech. 180, 21 (1987).

[25] J. Harting, J. Chin, M. Venturoli, and P. V. Coveney, Phil. Trans. R. Soc. A 363, 1895 (2005).

[26] H. Tanaka and T. Araki, Chem. Eng. Sci. 61, 2108 (2006).

[27] J. T. Padding and A. A. Louis, Phys. Rev. E 74, 031402 (2006).

[28] J. M. Yeomans, Physica A 369, 159 (2006). 